\title{
The Potential Roles of Superoxide Dismutase, Matrix-Metalloprotinase-9 and Interlukine -18 with the Prevalence and Progression of Type II Diabetes Mellitus
}

\author{
Shwan Ali Umar ${ }^{1}$, Shatha Rouf Moustafa ${ }^{2, *}$ \\ ${ }^{1}$ Ministry of Health, Zanco city, Erbil, Iraq \\ ${ }^{2}$ College of Pharmacy, Hawler Medical University, Havalan City, Erbil, Iraq \\ Email address: \\ shwann701@yahoo.com (S. A. Umar), shatha003@yahoo.com (S. R. Moustafa)
}

\section{To cite this article:}

Shwan Ali Umar, Shatha Rouf Moustafa. The Potential Roles of Superoxide Dismutase, Matrix-Metalloprotinase-9 and Interlukine -18 with the Prevalence and Progression of Type II Diabetes Mellitus. American Journal of Internal Medicine. Vol. 3, No. 3, 2015 , pp. $103-116$. doi: $10.11648 /$ j.ajim.20150303.14

\begin{abstract}
Background and Objectives: Type II diabetes mellitus is becoming the most important health problems. It is important to find new pathogenic pathways to provide opportunities for early diagnosis and targets for novel treatments. Aims: to investigate the effect of type II diabetes mellitus on serum levels super oxide dismutase as a potential biomarkers of oxidant /antioxidant imbalance, matrix-metaloprotinase-9, and interlukine -18 as an inflammatory biomarkers and estimated their relationships with prevalence and progression of disease, to find out the effects of other confounding factors severity of disease, gender, and age on the serum interested parameters levels, finally detected the correlation coefficient between the studied parameters. Methods: This study was designed to examine the associations between the parameters with the prevalence and progression of disease in 50 patients, and an equal number of matched age -gender healthy adults were also enrolled in this study as a control group. The hypothesis of the study stated that oxidant /antioxidant imbalance and inflammatory process influence the risk of adverse clinical outcomes are worthy for investigating. To elucidate this hypothesis, the parameters were investigated using enzyme linked immunosorbent assay. Statistical Analyses: All analyses were performed with SPSS version 18. Results: Patients showed a significant variations in the serum focused parameters levels. The level of superoxide dismutase in patient and control groups were $(301.684 \pm 97.041),(501.447 \pm 162.781)(\mathrm{ng} / \mathrm{ml})$ respectively. The level of matrixmetalloprotinase-9 in patient and control groups were $(2.632 \pm 1.745),(1.506 \pm 0.945) \mathrm{ng} / \mathrm{ml} \mathrm{respectively.} \mathrm{The} \mathrm{level} \mathrm{of}$ interlukine -18 in patients and control groups were $(93.996 \pm 21.297),(48.400 \pm 26.375)(\mathrm{pg} / \mathrm{ml})$ respectively. Conclusions: The prevalence of type II diabetes mellitus in the Erbil population constitutes a major health problem. Oxidative stress is increased in type II diabetes mellitus and this finding may explain the role of oxidant /antioxidants status imbalance leading to significant reduction in the serum superoxide dismutase level concomitant with increased inflammatory process leading to significant elevation of matrix- metaloptotinase- 9 and interlukine -18 , and these findings added a prognostic information to type II diabetes mellitus. Data demonstrated a highly significant association between the variation in the serum parameters levels with prevalence and progression of the disease. Accordingly, these parameters have been evaluated as potential tools for type II diabetes mellitus risk prediction. It can therefore be concluded that hyperglycemia influences the etiopathogenesis of type II diabetes mellitus.
\end{abstract}

Keywords: Diabetes Mellitus Type II, Superoxide dismutase, Matrixmetalloproteinase-9, Interlukine -18

\section{Introduction}

The term diabetes mellitus (DM) defines as a metabolic disorder of multiple etiology characterized by chronic hyperglycemia with disturbances of carbohydrate, fat and protein metabolism resulting from defects in insulin secretion, insulin action, or both.
Oxidative stress /antioxidant status imbalance and inflammatory process are involved in type II diabetes mellitus (T2DM) prevalence and progression. Oxidative stress (OS) plays a major role in the pathogenesis of T2DM [1]. Different studies have provided evidences that hyperglycemia induces OS and depleted antioxidant enzymes thus leading to increase free radical (FR) generation in type II diabetes [2-3]. 


\subsection{Diabetes Mellitus Type II and Oxidative Stress}

Oxidative stress is a phenomenon associated with pathogenic mechanisms of several diseases including DM which is defined as an imbalance between formation of FRs and reactive metabolites, so-called oxidants, and their elimination by protective mechanisms, referred to as antioxidative systems. The final result of this imbalance is the oxidation of important macromolecules, including proteins, lipids, carbohydrates and DNA [4 ]. There are convincing experimental and clinical evidences that the formation of reactive oxygen species (ROS) is increased in T2DM and that the onset of diabetes is closely associated with OS. Oxidative stress starts at early onset of diabetes mellitus and increases progressively [5]. Oxidative stress is the initial change induced by high glucose, followed by activation of other pathways that lead to cellular dysfunction and damage.

In recent years, much attention has been focused on the role of OS and it has been reported that OS may constitute the key and common event in the pathogenesis of secondary diabetic complications [6 ].

Growing evidence indicates that OS plays a pivotal role in insulin resistance, impaired insulin secretion and the development of both micro and macro vascular diabetic complications [7 ].

The ability of antioxidants to protect against the effects of hyperglycemia along with the clinical benefits often reported following antioxidant therapy, supports a causative role of OS in mediating and /or worsening these abnormalities.

\section{Superoxide Dismutase (EC 1.15.1.1)}

It is one of the cellular defense mechanism for OS [8]. It is the major antioxidant enzyme for superoxide removal, which converts superoxide into hydrogen peroxide $\left(\mathrm{H}_{2} \mathrm{O}_{2}\right)$ and molecular oxygen.

\subsection{Diabetes Mellitus Type II and Inflammatory Process}

The inflammatory response starts with signal recognition that may have an infectious or inflammatory origin, and the release of chemicals from tissues and migrating cells called mediators [9]. Inflammatory mediators appear to play a fundamental role in the initiation, prevalence and progression of T2DM and diabetic complications. Circulating cytokines are elevated in T2DM, obesity, and insulin resistance and play a central role in the pathogenesis of these disorders [10].

\subsubsection{Maltrixmetalloproteinase-9}

Matrix metalloproteinases (MMPs) are a family of zincbinding, endopeptidases proteolytic enzymes that normally remodeling and degrade extracellular matrix proteins and pathologically attack substrates as part of an inflammatory response [11]. Increased activity of MMPs has been reported in obesity, DM, arterial hypertension and dyslipidemia [12]. Their activity is regulated by tissue inhibitors of matrix metalloproteinase (TIMMPs) and also by other molecules, such as plasmin. Hyperglycemia and DM have been shown to produce more ROS, which activate MMP-9 via OS [13 ].

Cellular damage, increased extra cellular matrix (ECM) production and vascular dysfunction have all been implicated in the pathogenesis of vascular disease in type I and type II diabetes mellitus [14].

Extracellular matrix abnormalities are a structural hallmark in DM and fibrosis, characterized by ECM accumulation and angiogenesis. Hyperglycemia inhibits matrix degradation and affects the activities of the enzymes MMPs and their tissue inhibitors (TIMPs), as well as increases the synthesis of ECM components, including collagens, fibronectin [15 ].

\subsubsection{Interlukin -18}

Interleukins are regulatory proteins with ability to accelerate or inhibit inflammatory processes, interleukins may exhibit marked effects on the regulation of immune responses and the pathogenesis of a great variety of diseases. Interleukins-18 is expressed as a precursor, proinflammatory cytokine with multiple biologic functions, pro-IL18 which is inactive until cleaved by the enzyme caspase-1. Interleukins 18 stimulates T-helper-1 (TH-1) and mediated immune responses, so, it is an important mediator of innate immunity [16]. Furthermore, IL-18 leads to production of other proinflammatory molecules, including IL- 8 , IL-1 $\beta$, tumor necrosis factor- $\alpha$ (TNF- $\alpha)$, and intercellular adhesion molecule-1 [17 ] from mononuclear cells and macrophages. These molecules are known to increase in T2DM [18 ].

Interleukins-18 has also been suggested to be a potent proinflammatory cytokine that regulates autoimmune and inflammatory diseases [19]. Beside, it was published that increasing levels of circulating IL-18 have been reported to be closely associated with the components of metabolic syndrome components [20 ], hyperglycemia [21 ], T2DM [22 ], chronic complications of DM [23 ].

According to the above information, immunologic and inflammatory mechanisms play a significant roles in a development and progression of T2DM as well as Inflammation is considered as a conceivable etiologic mechanism for T2DM .

This case control study was designed to investigate the hypothesis that a combined intervention consisting of oxidative stress /antioxidant status imbalance concomitant with inflammation process interactions in patients with T2DM influence the risk of adverse clinical outcomes are worthy for investigating, to test the hypothesis the enzymatic antioxidant levels SOD as well as the inflammatory process by investigating IL-18 and MMP-9 were measured using enzyme linked immunosorbent assay (ELISA) as a potential predictors of T2DM on 50 patients with T2DM, and an equal numbers of matched age -gender apparently healthy adults were also enrolled in this study for comparing purposes as a control group. This would provide a better understanding of the role of ROS in T2DM that could lead to the development of new therapeutic strategies.

Therefore, because of impaired glucose metabolism leads to OS, so, the aim of this study was that to investigate the effect of T2DM on serum profile of SOD as a biomarker of oxidant /antioxidant status imbalance, MMP-9 and IL-18 as biomarkers of inflammatory processes and estimated their 
relationship with prevalence and progression of T2DM. In addition, to find out the effects of other confounding factors severity of disease, gender, and age on the serum levels of interested parameters, finally detected the correlation coefficient between the studied parameters.

\section{Patients and Methods}

This study designed to investigate the cooperation between oxidative stress, antioxidant status concomitant with inflammatory process with the prevalence of type II diabetes mellitus in 50 patients of both genders who were diagnosed at Diabetic Center (Al-Shahida Layla Qassim ), and an equal numbers of the matched age -gender were also enrolled in this study as a control group, both groups were completed the baseline questionnaire concerning several risk factors including history of smoking, physical activity, alcohol consumption, gender, age, other diseases, complains, family history, past medical history, and medication history.

This case control prospective study was performed at Hawler Medical University, College of Pharmacy in period between October 2013 to December 2014. The control group was confirmed to be normal by biochemical and hematological examinations. This study involved 95 participants, 50 patients with type II diabetes mellitus of both genders (25 men and 25 women) with a mean age at diagnosis $(55.98 \pm 8.277)$ years were enrolled in this study after exclusion of other diseases by clinical history, laboratory investigations and clinical examination, and for comparing purposes 45 matched age-gender apparently healthy adults were randomly selected as a control group, this group included ( 23) healthy men and (22) healthy women with a mean age $(55.9 \pm 7.5)$ years . All procedures were in accordance with the established ethical standards. Investigations: Biochemical ,hematological. All clinicopathological data of the patients were collected from the clinical files of the patients. Verbal informed consents were taken from all the participants before participation in the current study. The Ethics Committee of Medical Research at College of Pharmacy /Hawler Medical University approved the study protocol.

\subsection{Methods}

Diabetes mellitus was diagnosed on the basis of Water Health Organization (WHO) the fasting serum level of glucose $\geq 126 \mathrm{mg} / \mathrm{ml}$.

\subsubsection{Patients Selection}

The study carried out in 50 patients who attending in diabetic center ( Al-Shahyda Layla Qassim ), and 45 of matched age-gender apparently healthy adult as a control group were also enrolled in this study for comparing purposes. Patients with T2DM were diagnosed by specialist physician through clinical examination and confirmed by laboratory tests at diabetic center (Al-Shahida Layla Qassim).

\subsubsection{Sample Collection}

The Protocol of the Study: Ten $\mathrm{ml}$ of the fasting blood (overnight for 12-14 h) samples were collected from the vein of the participants without using tourniquet. The blood samples were left for 30 minutes for coagulation purpose, and then centrifuged for 15 minutes at 2500-3500 revolution per minutes (rpm). The sera of the patients were separated and divided into several parts and put them into several plastic plain tubes to do the biochemical tests of the current study. The sera of the patients were stored at $\left(-20 \mathrm{C}^{\circ}\right)$ till the day of the analysis within (1-2 months). The sera samples were prepared for measurement by warming the frozen sera at room temperature. The exclusion criteria were: Obesity, smoking, diseases (hypertension, cardiovascular disease, asthmatic patients, malignancies, muscular disease, renal disease, inflammatory diseases, chronic diseases), the current use of any medications, patients on lipid lowering drugs and antioxidant dietary supplements, vitamin supplements or minerals were also excluded from the current study. By depending on the physical, clinical examination, and laboratory investigations hematological, biochemical data to prevent the overlapping or interfering with the results of the present study because these diseases also have serum biochemical disturbances.

\subsection{Biochemical Determinations}

Oxidative stress /antioxidant status imbalance and inflammatory process were measured by quantification of SOD, MMP-9 and IL-18 using ELISA technique.

\subsection{Statistical Analysis}

Data were analyzed using the Statistical Patch for Social Sciences. The results of biochemical tests were expressed as mean \pm standard deviation, student t-test was applied to compare between two means. Post Hoc test (LSD) was used to show the significant difference between each two of three variables. Multiple regression was used to show the association between each of the biomarkers (as a dependent variables) and several independent variables. A (p) value of $\leq$ 0.05 was considered as statistical significance. Correlations between laboratory findings and continuous variables were evaluated using linear regression analysis.

\section{Results}

\subsection{Subject Characteristics}

The demographic and clinical characteristics of the patients and control groups were exhibited in (Table 1). This study included 95 participants of both genders , 50 of them were patients with T2DM which were divided into two groups poor control (35 patients with mean age $52.9 \pm 7.8$ ) and uncontrolled T2DM ( 15 patients with mean age $63.3 \pm$ $3)$.The reminders 45 were apparently healthy adults with mean age was $55.9 \pm 7.5$ 
Table 1. The demographic and clinical characteristics of the patients and control groups.

\begin{tabular}{|c|c|c|c|c|c|}
\hline & & $\mathbf{N}$ & Mean & \pm SD & $\mathbf{p}$ \\
\hline \multirow{4}{*}{ Age } & No DM & 45 & 55.933 & 7.500 & \multirow{4}{*}{$<0.001$} \\
\hline & Poor control & 35 & 52.857 & 7.845 & \\
\hline & Un-control & 15 & 63.267 & 3.011 & \\
\hline & Total & 95 & 55.958 & 7.877 & \\
\hline \multirow{4}{*}{ weight $/ \mathrm{kg}$} & No DM & 45 & 74.222 & 4.188 & \multirow{4}{*}{.168} \\
\hline & Poor control & 35 & 73.943 & 7.372 & \\
\hline & Un-control & 15 & 70.400 & 11.319 & \\
\hline & Total & 95 & 73.516 & 6.986 & \\
\hline \multirow{4}{*}{$\mathrm{HbA}_{1} \mathrm{C} \%$} & No DM & 45 & 5.281 & .183 & \multirow{4}{*}{$<0.001$} \\
\hline & Poor control & 35 & 8.386 & .323 & \\
\hline & Un-control & 15 & 10.487 & 1.499 & \\
\hline & Total & 95 & 7.247 & 2.097 & \\
\hline \multirow{4}{*}{ FBG } & No DM & 45 & 92.311 & 6.338 & \multirow{4}{*}{$<0.001$} \\
\hline & Poor control & 35 & 231.486 & 26.983 & \\
\hline & Un- control & 15 & 297.133 & 42.963 & \\
\hline & Total & 95 & 175.926 & 86.010 & \\
\hline \multirow{4}{*}{ MMP-9ng/ml } & No DM & 45 & 1.506 & .945 & \multirow{4}{*}{$<0.001$} \\
\hline & Poor control & 35 & 1.804 & .943 & \\
\hline & Un-control & 15 & 4.563 & 1.669 & \\
\hline & Total & 95 & 2.098 & 1.524 & \\
\hline \multirow{4}{*}{ IL-18 pg/ml } & No DM & 45 & 48.400 & 26.375 & \multirow{4}{*}{$<0.001$} \\
\hline & Poor control & 35 & 84.240 & 17.581 & \\
\hline & Un-control & 15 & 116.760 & 6.603 & \\
\hline & Total & 95 & 72.398 & 32.953 & \\
\hline \multirow{4}{*}{ SOD } & No DM & 45 & 501.447 & 162.781 & \multirow{4}{*}{$<0.001$} \\
\hline & Poor control & 35 & 357.320 & 43.149 & \\
\hline & Un-control & 15 & 171.867 & 51.409 & \\
\hline & Total & 95 & 396.308 & 165.428 & \\
\hline
\end{tabular}

\subsection{Effect of Type II Diabetes Mellitus on the Serum Levels of the Studied Parameters}

In patients with $\mathrm{T} 2 \mathrm{DM}$ there were a highly significant elevation in the serum levels of $\mathrm{HbA}_{1} \mathrm{C} \%$, glucose, MMp-9 and IL-18 as compared with the control group $\mathrm{p}<0.001$, while there was a highly significant reduction in the serum level of SOD in patients group as compared with the control group $\mathrm{p}<0.001$ (Table 2).

Table 2. Comparison between patients and control groups regarding the studied parameters.

\begin{tabular}{|c|c|c|c|c|c|c|}
\hline & Group & $\mathbf{N}$ & Mean & \pm SD & SE & $\mathbf{p}$ \\
\hline \multirow{2}{*}{ age } & patients & 50 & 55.980 & 8.277 & 1.171 & \multirow{2}{*}{.977} \\
\hline & Control & 45 & 55.933 & 7.500 & 1.118 & \\
\hline \multirow{2}{*}{ weight $/ \mathrm{kg}$} & patients & 50 & 72.880 & 8.775 & 1.241 & \multirow{2}{*}{.337} \\
\hline & Control & 45 & 74.222 & 4.188 & .624 & \\
\hline \multirow{2}{*}{$\mathrm{HbA}_{1} \mathrm{C} \%$} & patients & 50 & 9.016 & 1.288 & .182 & \multirow{2}{*}{$<0.001$} \\
\hline & Control & 45 & 5.281 & .183 & .027 & \\
\hline FBG & patients & 50 & 251.180 & 44.227 & 6.255 & $<0.001$ \\
\hline \multirow{2}{*}{ MMP-9 ng/ml } & patients & 50 & 2.632 & 1.745 & .247 & \multirow{2}{*}{$<0.001$} \\
\hline & Control & 45 & 1.506 & .945 & .141 & \\
\hline \multirow{2}{*}{ IL-18 pg/ml } & patients & 50 & 93.996 & 21.297 & 3.012 & \multirow{2}{*}{$<0.001$} \\
\hline & Control & 45 & 48.400 & 26.375 & 3.932 & \\
\hline \multirow{2}{*}{$\mathrm{SOD} \mathrm{ng} / \mathrm{ml}$} & patients & 50 & 301.684 & 97.041 & 13.724 & \multirow{2}{*}{$<0.001$} \\
\hline & Control & 45 & 501.447 & 162.781 & 24.266 & \\
\hline
\end{tabular}

3.3. The Effect of the Stage of Disease with Regard to Poor

\section{Controlled and Un-Controlled Type II Diabetes}

\section{Mellitus}

The serum levels of $\mathrm{HbA}_{1} \mathrm{C} \%$ and glucose were founded to be significantly higher in T2DM patients (un-control group) as compared with poor control and healthy subjects $\mathrm{p}<0.001$ (Table 1). On the other hand, there were a significant elevations in the serum levels of MMp-9 and IL-18 in T2DM patients (un-control group) as compared with poor control and healthy subjects $\mathrm{p}<0.001$ and there was a significant reduction in the serum level of SOD in T2DM patients (uncontrol group) as compared to poor control and healthy subjects $\mathrm{p}<0.001$.

\subsection{Gender-Effect}

There was a significant difference between men and women regarding MMP-9 $\mathrm{p}=0.049$, while there were no significant differences between men and women regarding SOD and IL-18 p > 0.05 (Table 3). 
Table 3. Comparison between men and women regarding the studied parameters in patient group.

\begin{tabular}{llllll}
\hline & Sex & N & Mean & SD & p \\
\hline \multirow{2}{*}{ MMP-9ng/ml } & Men & 25 & 2.512 & 1.840 & .049 \\
& Women & 25 & 1.861 & 1.070 & \\
\multirow{2}{*}{ IL-18 pg/ml } & Men & 25 & 70.906 & 36.060 & .665 \\
\multirow{2}{*}{ SOD } & Women & 25 & 73.858 & 29.912 & \\
& Men & 25 & 367.226 & 128.068 & .090 \\
\hline
\end{tabular}

\subsection{Age-Effect}

There were a moderate significant negative correlations between SOD with age $\mathrm{r}=-0.676 ; \mathrm{P}=0.001$, there were a moderate significant positive correlations between MMP-9 with age $\mathrm{r}=0.501 ; \mathrm{p}=0.001$, there were a moderate significant positive correlations between IL-18 with age $\mathrm{r}=0.613$; $\mathrm{p}=0.001$, there were a moderate significant positive correlations between $\mathrm{HbA}_{1} \mathrm{C}$ with age $\mathrm{r}=0.613 ; \mathrm{p}=0.001$, there were a moderate significant positive correlations between FBG with age $\mathrm{r}=0.550 ; \mathrm{p}=0.001$ ( Table 4).

\subsection{Correlation Coefficient}

\subsubsection{Correlation Coefficient Between Studied Parameters in Patient Group}

The correlation coefficient between all serum levels of studied parameters in patients group were presented in (Table 4).

There were a strong significant positive correlations between $\mathrm{HbA}_{1} \mathrm{C}$ with $\mathrm{FBG} \mathrm{r}=0.842, \mathrm{p}=0.001$; there were a strong significant positive correlations between $\mathrm{HbA}_{1} \mathrm{C}$ with MMp-9 $r=0.757, p=0.001$; there were a strong significant positive correlations between $\mathrm{HbA}_{1} \mathrm{C}$ with $\mathrm{IL}-18 \mathrm{r}=0.764$, $\mathrm{p}=0.001$; there were a strong significant negative correlations between $\mathrm{HbA}_{1} \mathrm{C}$ with SOD $\mathrm{r}=-0.883, \mathrm{p}=0.001$; there were a moderate significant positive correlations between $\mathrm{HbA}_{1} \mathrm{C}$ with age $\mathrm{r}=0.613, \mathrm{p}=0.001$. Moreover, there were a moderate significant positive correlations between FBG with MMp-9 $\mathrm{r}=0.561, \mathrm{p}=0.001$; there were a strong significant positive correlations between FBG with IL-18 $\mathrm{r}=0.818$, $\mathrm{p}=0.001$; there were a strong significant negative correlations between FBG with SOD $\mathrm{r}=-0.848, \mathrm{p}=0.001$; there were a moderate significant positive correlations between FBG with age $\mathrm{r}=0.550, \mathrm{p}=0.001$. In addition, there were a weak significant positive correlations between MMP-9 with IL-18 $\mathrm{r}=0.452, \mathrm{p}=0.001$; there were a strong significant negative correlations between MMP-9 with SOD $\mathrm{r}=-701, \mathrm{P}=0.001$; there were a moderate significant positive correlations between MMP-9 with age $r=0.501 \mathrm{p}=0.001$. Furthermore, there were a strong significant negative correlations between IL-18 with SOD $r=-0.889, p=0.001$; there were a moderate significant positive correlations between IL-18 with age $\mathrm{r}=0.613, \mathrm{p}=0.001$. Finally, there were a moderate significant negative correlations between SOD with age $\mathrm{r}=-0.676$, $\mathrm{P}=0.001$.

Table 4. The correlation coefficient between All Studied Parameters in patients group

\begin{tabular}{|c|c|c|c|c|c|c|c|c|}
\hline & & weight/kg & $\mathrm{HbA}_{1} \mathrm{C} \%$ & FBG & MMP-9ng/ml & IL-18 pg/ml & SOD & age \\
\hline \multirow{3}{*}{ weight $/ \mathrm{kg}$} & Pearson Correlation & 1 & -.060 & -.080 & -.108 & -.102 & .114 & -.068 \\
\hline & Sig. (2-tailed) & & 679 & .580 & .455 & .482 & .432 & .640 \\
\hline & $\mathrm{N}$ & 50 & 50 & 50 & 50 & 50 & 50 & 50 \\
\hline \multirow{3}{*}{$\mathrm{HbA}_{1} \mathrm{C} \%$} & Pearson Correlation & -.060 & 1 & $.842^{* *}$ & $.757^{* *}$ & $.764^{* *}$ & $-.883^{* *}$ & $.613^{* *}$ \\
\hline & Sig. (2-tailed) & .679 & & .000 & .000 & .000 & .000 & .000 \\
\hline & $\mathrm{N}$ & 50 & 50 & 50 & 50 & 50 & 50 & 50 \\
\hline \multirow[t]{2}{*}{ FBG } & Sig. (2-tailed) & .580 & .000 & & .000 & .000 & .000 & .000 \\
\hline & $\mathrm{N}$ & 50 & 50 & 50 & 50 & 50 & 50 & 50 \\
\hline \multirow{3}{*}{ MMP-9ng/ml } & Pearson Correlation & -.108 & $.757^{* *}$ & $.561^{* *}$ & 1 & $.492^{* *}$ & $-.701^{* *}$ & $.501^{* *}$ \\
\hline & Sig. (2-tailed) & .455 & .000 & .000 & & .000 & .000 & .000 \\
\hline & $\mathrm{N}$ & 50 & 50 & 50 & 50 & 50 & 50 & 50 \\
\hline \multirow[b]{2}{*}{ IL-18 pg/ml } & Pearson Correlation & -.102 & $.764^{* *}$ & $.818^{* *}$ & $.492^{* *}$ & 1 & $-.889^{* *}$ & $.613^{* *}$ \\
\hline & Sig. (2-tailed) & .482 & .000 & .000 & .000 & & .000 & .000 \\
\hline \multirow{3}{*}{ SOD } & Pearson Correlation & .114 & $-.883^{* *}$ & $-.848^{* *}$ & $-.701^{* *}$ & $-.889^{* *}$ & 1 & $-.676^{* *}$ \\
\hline & Sig. (2-tailed) & .432 & .000 & .000 & .000 & .000 & & .000 \\
\hline & $\mathrm{N}$ & 50 & 50 & 50 & 50 & 50 & 50 & 50 \\
\hline \multirow{3}{*}{ age } & Pearson Correlation & -.068 & $.613^{* *}$ & $.550^{* *}$ & $.501^{* *}$ & $.613^{* *}$ & $-.676^{* *}$ & 1 \\
\hline & Sig. (2-tailed) & .640 & .000 & .000 & .000 & .000 & .000 & \\
\hline & $\mathrm{N}$ & 50 & 50 & 50 & 50 & 50 & 50 & 50 \\
\hline
\end{tabular}

**. Correlation is significant at the 0.01 level (2-tailed). 


\subsubsection{Correlation Coefficient Between Studied Parameters in Control Group}

The correlation coefficient between all serum levels of studied parameters in patients group were presented in (Table 5). There were a moderate positive significant correlation between $\mathrm{HbA}_{1} \mathrm{C}$ with MMp-9 $\mathrm{r}=0.516, \mathrm{p}=0.001$; there were a strong negative significant correlation between $\mathrm{HbA}_{1} \mathrm{C}$ with SOD $r=-0.702, p=0.001$; there were a moderate positive significant correlation between $\mathrm{HbA}_{1} \mathrm{C}$ with age $\mathrm{r}=0.419$, $\mathrm{p}=0.004$.

Table 5. The correlation coefficient between all studied parameters in control group.

\begin{tabular}{|c|c|c|c|c|c|c|c|c|}
\hline & & weight/kg & $\mathrm{HbA}_{1} \mathrm{C} \%$ & FBG & MMP-9ng/ml & IL-18 pg/ml & SOD & age \\
\hline \multirow{3}{*}{ weight $/ \mathrm{kg}$} & Pearson Correlation & 1 & -.110 & .205 & .034 & .217 & .175 & .168 \\
\hline & Sig. (2-tailed) & & .472 & .178 & .827 & .152 & .251 & .269 \\
\hline & $\mathrm{N}$ & 45 & 45 & 45 & 45 & 45 & 45 & 45 \\
\hline \multirow{3}{*}{$\mathrm{HbA}_{1} \mathrm{C} \%$} & Pearson Correlation & -.110 & 1 & -.163 & $.516^{* *}$ & -.017 & $-.702^{* *}$ & $.419^{* *}$ \\
\hline & Sig. (2-tailed) & .472 & & .285 & .000 & .913 & .000 & .004 \\
\hline & $\mathrm{N}$ & 45 & 45 & 45 & 45 & 45 & 45 & 45 \\
\hline \multirow{3}{*}{ FBG } & Pearson Correlation & .205 & -.163 & 1 & -.003 & .171 & .025 & .272 \\
\hline & Sig. (2-tailed) & .178 & .285 & & .986 & .263 & .872 & .071 \\
\hline & $\mathrm{N}$ & 45 & 45 & 45 & 45 & 45 & 45 & 45 \\
\hline \multirow{3}{*}{ MMP-9ng/ml } & Pearson Correlation & .034 & $.516^{* *}$ & -.003 & 1 & -.127 & -.255 & .116 \\
\hline & Sig. (2-tailed) & .827 & .000 & .986 & & .407 & .091 & .450 \\
\hline & $\mathrm{N}$ & 45 & 45 & 45 & 45 & 45 & 45 & 45 \\
\hline \multirow{3}{*}{ IL-18 pg/ml } & Pearson Correlation & .217 & -.017 & .171 & -.127 & 1 & .037 & .016 \\
\hline & Sig. (2-tailed) & .152 & .913 & .263 & .407 & & .809 & .915 \\
\hline & $\mathrm{N}$ & 45 & 45 & 45 & 45 & 45 & 45 & 45 \\
\hline \multirow{3}{*}{ SOD } & Pearson Correlation & .175 & $-.702^{* *}$ & .025 & -.255 & .037 & 1 & -.270 \\
\hline & Sig. (2-tailed) & .251 & .000 & .872 & .091 & .809 & & .073 \\
\hline & $\mathrm{N}$ & 45 & 45 & 45 & 45 & 45 & 45 & 45 \\
\hline \multirow{3}{*}{ age } & Pearson Correlation & .168 & $.419^{* *}$ & .272 & .116 & .016 & -.270 & 1 \\
\hline & Sig. (2-tailed) & .269 & .004 & .071 & .450 & .915 & .073 & \\
\hline & $\mathrm{N}$ & 45 & 45 & 45 & 45 & 45 & 45 & 45 \\
\hline
\end{tabular}

**. Correlation is significant at the 0.01 level (2-tailed).

\section{Discussion}

\subsection{General View}

This study was designed to identify whether oxidative stress / antioxidant status imbalance and inflammatory process occurred in patients with T2DM as an events of disease prevalence and progression, so, this study was aimed to address the potential role of antioxidant enzyme superoxide dismutase, in addition to investigate the role of matrix metalloproteinase- 9 in the inhibition of matrix metalloprotein degradation by high glucose serum levels and to measure the serum level of IL-18 as a marker of inflammation process in the serum levels of all participants .

This study has shown that antioxidant marker SOD concomitant with circulating inflammatory markers MMP-9 and IL-18 can be used as a useful biomarkers for distinguishing patients with T2DM from normal subjects.

Data were consistent with the hypothesis of the present study, that there were a highly significant association between significant reduction in the serum level of SOD and significant elevation in the serum levels of MMP-9 and IL-18 with prevalence and progression of T2DM.

This study has indicated the presence of OS, inflammatory process and diminished antioxidant status in T2DM patients.

An increasing evidence suggesting of the possible mechanisms through which OS exerts a regulatory role in T2DM incidence, development and progression.

This study investigated the major antioxidant enzyme SOD and inflammatory parameters MMP-9 and IL-18 in order to obtain a comprehensive view of the type II diabetic patients, the serum levels of focused parameters were correlated with other confounding factors such as stage of the disease, gender, age, and finally detected the correlation coefficient between all studied parameters.

The study was aimed to determine the predictive prognostic roles of these biomarkers for disease outcome. So, the purpose of this study was to investigate the roles of these interested parameters in prevalence and progression of T2DM.

The values of all the parameters were statistically compared with apparently healthy adults as a control group. The results suggested that the lowered serum antioxidant level may be as a consequence result of the OS of the disease.

The present study was designed to evaluate the activity of antioxidant enzyme SOD and inflammatory markers MMp-9 and IL-18 in type II diabetic patients and control group, and to examine the relationship between the measured parameters 
to define the potential clinical relevance of identifying alterations affecting SOD, IL-18 and MMP-9 in type II diabetic patients.

The demographic and clinical characteristics of the patients and control groups were exhibited in (Table 1). This study included 95 participants of both genders, 50 of them were patients with T2DM which were divided into two groups poor control (35 patients with mean age $52.9 \pm 7.8$ ) and uncontrolled T2DM (15 patients with mean age $63.3 \pm 3$ ). The reminders 45 were apparently healthy adults with mean age was $55.9 \pm 7.5$.

\subsection{Effect of the Type II Diabetes Mellitus on the Serum Levels of Studied Parameters}

\subsubsection{Anti-Oxidant Status Marker Superoxide Dismutase}

The hypothesis was supported by the current findings that the levels of ROS increase in patients with T2DM and thereby cause depletion of antioxidant enzyme SOD in patients as compared with the control group .

In patients with T2DM, data indicated that there was a highly significant reduction in the serum level of SOD in patients group as compared with the control group $\mathrm{p}<0.001$ (Table 2). In addition, data of the present study supported a possibility that hyperglycemia might be related to decreased activity of SOD, and given a strong negative correlation between serum level of SOD and $\mathrm{a} \mathrm{HbA}_{1} \mathrm{C} \mathrm{r}=-0.883$, $\mathrm{p}=0.001$ (Table 4), as much as $\mathrm{HbA}_{1} \mathrm{C}$ increase SOD decrease which showed more OS.

There is a considerable evidence indicating that hyperglycemia may interfere with natural defence of the antioxidant system, in addition to increase the production of FRs [24] as well as several studies have reported lower concentration of enzymatic antioxidants in type II diabetes [2,25-27].

The current finding and other result revealed the implication of OS in the pathogenesis of T2DM and alteration in antioxidant enzymes [28]. Enhanced OS and changes in antioxidant capacity, observed in both clinical and experimental diabetes mellitus, are believed to be the etiology of chronic diabetic complications [29].

To evaluate the antioxidant status in patients with T2DM in Erbilian population, the antioxidant status was assessed by determining the enzyme SOD. The present study examined the antioxidant status marker SOD in the T2DM patients as compared to normoglycemic subjects. The significant reduction in serum level of SOD which was strongly associated with incidence of T2DM and might represent a useful prognostic marker in T2DM.

It was published that, superoxide dismutase is believed to be the most important among the clinically assessable enzymes involved in the OS [30].

This result may be explained an increased OS due to an overproduction of ROS radicals in patients with T2DM, and is paralleled by a significant reduction of the relevant enzymes involved, particularly SOD.

The study of the current study was consistent with the previous findings[24,27,31-34].
The above result was an indicator of decreased in the protective antioxidant mechanism SOD because of an increased production of FRs and ROS. According to the result of the current study, SOD has been considered as a reliable marker of oxidative stress / antioxidant status imbalance and has a potential role in the progression and development of T2DM and it is associated with incidence of this disease.

Accordingly, data of the current study confirmed and supported the hypothesis of previous researchers that OS plays a major role in the pathogenesis of T2DM which is a widely accepted contributor in the prevalence and progression of diabetes and its complications [6].

It has been reported that diabetic patients have significant defects of antioxidant protections and generation of ROS (oxidative stress) which may play an important role in the etiology of diabetic complications [35]. So, according to the current result that the significant reduction in the serum levels of SOD are thought to be related with the etiology of T2DM.

The diminished levels of antioxidant defense mechanism in the patients with T2DM can be accounted by two theories. In the first, circulating antioxidant enzymes may have been depleted in the attempt to counteract the DNA, lipid and protein damage. On another hand, the elevated DNA, lipid and protein oxidation may have occurred as a result of a weakened defense mechanism [36].

The scientific explanation for significant reduction in the serum level of SOD of the current study was that, SOD enzyme is a part of the first line of defence against FRs, it is expected that the activity of SOD may be affected by OS before the other antioxidant enzymes [24], decrease in SOD levels can result not only an increase in the superoxide-free radical but also an elevation of other ROS and intensification of lipid peroxidation processes in diabetes [27], in addition, there is an evidence to show that hyperglycemia is accompanied by the loss of $\mathrm{Cu}^{2+}$ as a result of osmotic diuresis, $\mathrm{Cu}^{2+}$ is an essential cofactor in SOD activity, furthermore, SOD is inactivated by glycosylation in erythrocytes [24]. Moreover, the decreasing of SOD activity observed with progression of diabetes could be due to glycation of the enzyme which occurs in the diabetic state [37]. Beside, the reduction in SOD activity could also be due to accumulation of HO FR in diabetic tissues which has been shown to inhibit SOD[38]. Furthermore, in diabetic patients, the autoxidation of glucose results in the formation of $\mathrm{H}_{2} \mathrm{O}_{2}$ which inactivates SOD [39]. Thus, increased glycation in diabetic patients and subsequent reactions of proteins may affect amino acids close to the active sites of the enzyme or disturb the stereochemical configuration and causes functional and structural variation in the molecule. This finding could also explain the progressive decrease in SOD in later stages of the diabetes.

Protective role of scavenger enzymes SOD may be deteriorated by OS. This result was suggested that diabetesinduced OS and played a key role in the prevalence and progression of T2DM . 
In contrast, to the result of the current study which was done by $[32,40]$ who reported that, the activity of SOD was significantly higher in type II diabetic patients as compared with healthy subjects. These differences could be due to the method of determination and units of expression or the stage of disease or life style variation.

\subsubsection{Inflammatory Parameter Matrixmetalloprotinase-9}

The inflammatory hypothesis of T2DM proposed a relationship between inflammation and T2DM. The suggestion of this study was based upon the observation that inflammation has been implicated in the pathogenesis and pathophysiology of T2DM. High glucose concentration inhibits matrix degradation and affects the activities of the enzymes, the matrix metalloproteinases (MMPs) and their tissue inhibitors (TIMPs) [41]. As MMP-9 expression can be promoted by OS and hyperglycaemia that characterizes of T2DM.

The result of the current study demonstrated that there was a significant elevation in the serum level of MMP-9 as compared with the control group $\mathrm{p}<0.001$ (Table 2 ).

The result of the present study was concordant with previous findings [42-49], who reported a significant increase in serum level of MMP-9 as compared the control group.

This study also highlight the role of MMP-9 to predict the development and progression of T2DM as well as supported the association between significant elevation of serum level of MMp-9 with incidence of T2DM.

The explanation of increased expression of MMP-9 in T2DM was that hyperglycemia directly or indirectly (e.g., via oxidative stress or advanced glycation products) might increase MMP expression and activity in large vessels [50], there is an accumulating evidence indicated that OS may play an important role in the pathogenesis of T2DM and MMP-9 is activated by ROS, and their expression seems to be regulated by OS [51]. Furthermore, OS appears to play a primary role in glucose-induced MMP-9 activity, suggesting a possible beneficial effect of antioxidant therapy in T2DM.

So, according to the current finding, it was concluded that high glucose level, suggesting that OS is involved in MMP-9 induction by hyperglycemia.

Data of the present study supported a possibility that hyperglycemia might be related to increased activity of MMP-9, and given the strong positive correlation between serum MMP-9 concentrations and $\mathrm{HbA}_{1} \mathrm{C} \mathrm{r}=0.757, \mathrm{p}=0.001$ (Table 4 ), in addition there were a moderate significant correlation between FBG with MMP-9 r=0.561, $\mathrm{p}=0.001$.

In contrary to the expectations of the current study, [52-54] have demonstrated a lower concentrations of MMP-9 in subjects with T2DM .While, [55] recognized that the plasma concentration of MMP-9 was not different between diabetic and non-diabetic participants.

\subsubsection{Interlukine -18}

This study evaluated the effects of T2DM on inflammatory marker IL-18. It is widely recognized that T2DM is associated with low-grade chronic inflammation [56]. It has been indicated that increased inflammation is an independent risk factor for the development of T2DM [57].

This study was performed to investigate the role of inflammatory marker IL-18 as a potential predictors of T2DM. There was a significant elevation in the serum level of IL-18 as compared with the control group $\mathrm{p}<0.001$ (Table 2). So, according to the current finding, it was concluded that high glucose level, suggesting that inflammatory process is involved in IL-18 induction by hyperglycemia.

Indeed, there was a significant interaction between elevated glucose and serum IL-18 level. Data of the present study supported a possibility that hyperglycaemia might be related to increased activity of IL-18, and given a strong significant positive correlation between $\mathrm{HbA}_{1} \mathrm{C}$ and IL-18 $\mathrm{r}=$ $0.76, \mathrm{p}<0.001$ (Table 4), as much as $\mathrm{HbA}_{1} \mathrm{C}$ increased the serum level of IL-18 increased, in addition there were a strong significant association between FBG and IL-18 $\mathrm{r}=0.818, \mathrm{p}=0.001$ (Table 4).

IL-18 has been shown to predict the development of T2DM [58]. On the other hand, experimental hyperglycemia has been shown to increase concentrations of IL-18 [21].

Several studies have provided further support to the inflammation / T2DM association by demonstrating higher serum levels of IL-18 among patients with T2DM.

As it was shown in (Table 2), the result of the current study was concordant with many previous findings [59-64] who reported that significant elevation in the serum levels of IL-18 in patients with T2DM as compared with control group.

This finding has suggested that IL-18 might be involved in the pathogenesis of the T2DM. So, data of the current study supported a role of inflammation in the pathogenesis and pathophysiology of T2DM.

Accordingly, the result of the present study added to the mounting evidence that T2DM might be regarded as a chronic low-grade inflammatory state.

The result of the this study supported the hypothesis that inflammatory mechanisms might play an important role in the incidence, development and progression of T2DM .

In the line with the current result, IL-18 has been shown to predict the development of type II diabetes mellitus [58].

In this case control prospective study the elevated levels of IL-18 have been shown to predict the development and progression of T2DM and there was an association between significant elevation of serum level of IL-18 with incidence of T2DM .

Recent studies have suggested that an inflammatory mechanism mediated by macrophages may play important roles in the pathogenesis of T2DM and have shown that inflammation is a key process in the development of diabetes mellitus and diabetic complications.

The mechanisms for elevation of serum IL-18 levels in T2DM remain unclear, although OS is a candidate [65 ].

Interleukin-18 is a potent proinflammatory cytokine that has been reported to be associated with the development of T2DM [58,66], and recently involved in the pathogenesis of DM. Elevated glucose was an independent predictor of events for all inflammatory markers, the event rates were higher in patients with elevated glucose . 


\subsection{Effect of Stage of Disease with Regard to Poor Controlled and Un-Controlled Type II Diabetes Mellitus}

The serum levels of $\mathrm{HbA}_{1} \mathrm{C} \%$ and glucose were founded to be significantly higher in T2DM patients (un-control group) as compared with poor control and healthy subjects $(\mathrm{p}<0.001)$ (Table 1). On the other hand, there were a significant elevations in the serum levels of MMp-9 and IL-18 in T2DM patients (un-control group) as compared to poor control and healthy subjects $(p<0.001)$ and regarding SOD, there was a significant reduction in the serum level of SOD in T2DM patients (un-controlled diabetic patients group as compared to poor control and healthy subjects ( Table 1) ( $\mathrm{p}<0.001)$.

This parameter (SOD) indicated that, an OS is more during uncontrolled stage of diabetes and this result was agreeable with the previous findings [27] .

\subsection{Gender-Effect}

The comparison between men and women regarding the studied parameters in patients group was presented in (Table 3 ), there was a significant difference between men and women regarding MMP- $9 \mathrm{p}=0.049$, while there were no significant differences between men and women regarding SOD and IL-18 $\mathrm{p}>0.05$.

Similarly, the study of [27] has examined the relationship between gender and oxidative stress / antioxidant status, in diabetic condition, SOD level was slightly decreased but no significant difference was found in diabetic men compared with diabetic women. The study of [27], clearly showed that diabetic patients, irrespective of the gender, were exposed to an increased OS via lipid peroxidation.

There are a number of reasons for discrepancies between studies. The external environment is also a significant contributor to OS. Other factors like body composition, smoking status, diet, physical activity level, and the strength of defense mechanism are also important.

\subsection{Age-Factor}

\subsubsection{Effect of Aging on Glucose Serum Level in Type II Diabetes Mellitus}

Diabetes mellitus type 2 progressively increases with age. In 2005, the prevalence of DM in the United States was estimated to be $0.22 \%$ for persons $<20$ years of age and $9.6 \%$ in those $>20$ years of age. In individuals $>60$ years old, the prevalence of DM was $20 \%$.

Type II diabetes mellitus is a common burden in the elderly [67]. There are a number of changes that occur as individuals aged. Aging is associated with alterations in body composition, which has implications in the development of insulin resistance and diabetes .

These information supported the result of the current study that elderly individuals are at a high risk to develop T2DM. The average age of patients of the current study at diagnosis was $55.98 \pm 8.277$ (Table 2). In addition, in accordance with the current study [68] reported that more than $80 \%$ of the type II diabetics were over the age of 40. Data of [69] suggested that an increased incidence of T2DM in the elderly could be due to alterations in the homeostatic mechanisms of TNF- $\alpha$, IL- 6 , and CRP levels, and that low-grade systemic inflammation occurs in T2DM.

\subsubsection{Effect of Aging on Studied Parameters}

In patients group, (Table 4) exhibited that there were a moderate significant negative correlations between SOD with age $\mathrm{r}=-0.676 ; \mathrm{P}=0.001$, SOD activity decreases with advancing age, this finding was concordant with the concept that in advanced age there is more OS and more FRs generation which lead to inhibit the activity of antioxidant defense mechanism as well as this finding was concordant with previous result of [70]. While [27] reported that, SOD lowers in diabetic age group $\geq 50$ yrs compared with age groups 30-39 and 40-49 yrs. So, the results of [27] indicated that OS impacts the aging process that may be caused by a number of factors including increased FR generation, decreased antioxidant system, or a decreased removal or repair.

Although the exact mechanisms are not known, it is assumed that $\mathrm{OS}$ is an important factor in the aging process and the individuals who are more resistant to the accumulation of oxidative damage will live longer. As the prevalence increases with age, the lifetime risk of developing diabetes is around $10 \%$ [71].

In addition, there were a moderate significant positive correlations between MMP-9 with age $\mathrm{r}=0.501 ; \mathrm{p}=0.001$, there were a moderate significant positive correlations between IL-18 with age $\mathrm{r}=0.613$; $\mathrm{p}=0.001$, there were a moderate significant positive correlations between $\mathrm{HbA}_{1} \mathrm{C}$ with age $\mathrm{r}=0.613 ; \mathrm{p}=0.001$, there were a moderate significant positive correlations between FBG with age $\mathrm{r}=0.550 ; \mathrm{p}=0.001$ ( Table 4).

In control group (Table 5 ) revealed that there was a negative weak correlation between Age and SOD ( $\mathrm{r}=-$ $0.270, \mathrm{p}=0.073$ ). This finding was consistent with previous study of [27] who published that, no significant correlation was found between antioxidant enzyme SOD activity and various age groups in non-diabetic healthy persons.

\subsection{Correlation Coefficient}

The correlation coefficient between all serum levels of studied parameters in patients group were presented in (Table 4). There were a strong significant positive correlations between $\mathrm{HbA}_{1} \mathrm{C}$ with $\mathrm{FBG} \mathrm{r}=0.842, \mathrm{p}=0.001$; there were a strong significant positive correlations between $\mathrm{HbA}_{1} \mathrm{C}$ with MMp-9 $r=0.757, p=0.001$; there were a strong significant positive correlations between $\mathrm{HbA}_{1} \mathrm{C}$ with $\mathrm{IL}-18 \mathrm{r}=0.764$, $\mathrm{p}=0.001$; there were a strong significant negative correlations between $\mathrm{HbA}_{1} \mathrm{C}$ with SOD $\mathrm{r}=-0.883, \mathrm{p}=0.001$; there were a moderate significant positive correlations between $\mathrm{HbA}_{1} \mathrm{C}$ with age $r=0.613, p=0.001$. Moreover, there were a moderate significant positive correlations between FBG with MMp-9 $r=0.561, p=0.001$; there were a strong significant positive correlations between FBG with IL-18 $\mathrm{r}=0.818$, $\mathrm{p}=0.001$; there were a strong significant negative correlations 
between FBG with SOD $r=-0.848, p=0.001$; there were a moderate significant positive correlations between FBG with age $\mathrm{r}=0.550, \mathrm{p}=0.001$. In addition, there were a weak significant positive correlations between MMP-9 with IL-18 $\mathrm{r}=0.452, \mathrm{p}=0.001$; there were a strong significant negative correlations between MMP-9 with SOD $\mathrm{r}=-701, \mathrm{P}=0.001$; there were a moderate significant positive correlations between MMP-9 with age $r=0.501 \mathrm{p}=0.001$. Furthermore, there were a strong significant negative correlations between IL-18 with SOD $\mathrm{r}=-0.889, \mathrm{p}=0.001$; there were a moderate significant positive correlations between IL-18 with age $\mathrm{r}=0.613, \mathrm{p}=0.001$. Finally, there were a moderate significant negative correlations between SOD with age $\mathrm{r}=-0.676$, $\mathrm{P}=0.001$.

There was a significant strong negative correlation between FBG and SOD ( $r=-0.848, p=0.001)$. Moreover there was a significant strong negative correlation between SOD and $\mathrm{HbA}_{1} \mathrm{C} \mathrm{r}=-0.883, \mathrm{p}=0.001$. In addition, there was a strong significant positive correlation between FBG and $\mathrm{HbA}_{1} \mathrm{C} \mathrm{r}=0.842, \mathrm{p}<0.001$ (Table 4).

While the study of [72] who reported that, in spite of low activities of SOD in T2DM, a negative, but not significant correlation between the activities of the enzyme and levels of glycated hemoglobin, fasting blood glucose.

Strengths of the study: The presented study has a strength that, the current study was the first prospective study in Erbil population that was performed to investigate the combined effects of oxidative /antioxidant status imbalance and inflammatory process by investigating SOD, MMP-9 and IL18 markers in T2DM risk prediction.

Accordingly, these results have provided the first evidence of a close association of serum SOD, MMP-9 and IL-18 levels in combination pattern with the prevalence and progression of T2DM, the significant reduction in serum level of SOD and the significant elevation of MMp-9 and IL18 in combination pattern, have been proposed as the most interesting findings to explain the pathophysiology of T2DM.

The most significant novelty in the present study is the presence of a correlation between the patient biological parameters such as antioxidant enzyme activity SOD, MMP9 and IL-18 with the prevalence and progression of T2DM.

Clinical utility and implication of this study: The social and economic costs of T2DM are enormous, for health care services and through loss of productivity. The importance of this study is therefore to supply an update current thinking about T2DM to improve the physical health of the patients.

The therapeutic use of antioxidants might be a useful approach. However, conventional antioxidants are effective because these compounds neutralize reactive oxygen molecules as well as the pharmacologic targeting of these parameters might provide an effective means to simultaneously control T2DM.

These analyses may provide new insight on the mechanisms through which OS may affect the clinical symptoms and disease progression.

The growing knowledge and better understanding of the role of the inflammatory pathway in the T2DM will represent an essential therapeutic opportunity for the production of new strategies that can be used successfully into clinical applications for the therapy of this disease. Moreover, this finding suggested the possibility of a potential effect of inflammation in T2DM risk prediction.

This study revealed that inflammatory markers MMP-9 and IL-18 added a potential prognostic information regarding T2DM. Indeed, there was a significant interaction between elevated glucose with MMP-9 and IL-18 which have been shown to predict the prevalence and progression of T2DM. The results of the current study point out to a more generalized concept with increased susceptibility to inflammation in the presence of hyperglycemia.

Data of the current study warrant further investigation with adequate clinical trials to test the hypothesis that supplementation of antioxidant agents may prevent /protect the patients from oxidative stress.

Alterations in the serum levels of these parameters contributed with T2DM prevalence, progression and are likely to provide relevant prognostic information to assist in the management of T2DM .

The main purpose of the research is that it may publish something which will help either directly or indirectly - the patients who look after.

\section{Conclusion}

This case control study was demonstrated a significant reduction in the serum level of SOD concomitant with significant elevations in the serum levels of MMP-9 and IL18 as compared with the control group. The main findings in this study was strongly predicted T2DM and were associated with the prevalence and progression of T2DM.

Hyperglycemia induces the overproduction of oxygen free radicals and consequently decreased the activity of SOD. The results of the current study revealed that OS in T2DM can be accelerated not only due to increased production of ROS caused by hyperglycemia but also by reduced ability of antioxidant defense system SOD. The findings of the present study suggested that T2DM was altered the metabolic state of oxidation-reduction and that it was convenient to give therapeutic interventions with antioxidants.

It is concluded on the basis of the present study that FRs are implicated in the etiology of T2DM and that enzymatic antioxidant SOD had a major role in preventing cellular oxidative damage.

Significant reduction in serum levels of SOD in type II diabetic patients indicated depletion of antioxidant mechanism and prognosis of OS. Therefore, estimations of this antioxidant enzyme SOD might be used as marker in the management of glycemic control and the development of diabetic complications.

Moreover, this finding suggested the possibility of potential effect of inflammation in T2DM risk prediction. This study suggested that inflammation was a key process in the development of T2DM. This finding revealed a potential role of hyperglycemia on inflammation which was presented 
by significant elevation in the serum levels of MMP-9 and IL-18 in patients with T2DM as compared with the control group.

Matrix metalloproteinase -9 activity is increased in T2DM, in part because of enhanced OS which plays an important role in the prevalence of T2DM. Accordingly, this study suggested that OS is involved in MMP-9 induction by hyperglycemia. So , OS appears to play a primary role in glucose-induced MMP-9 activity, suggesting a possible beneficial effect of antioxidant therapy in the complications of T2DM.

This novel mechanism of redox-sensitive MMP-9 expression by hyperglycemia may provide a rationale for antioxidant therapy to modulate T2DM. It can therefore be concluded that hyperglycemia influences the etiopathogenesis of T2DM .

The inflammatory molecule IL-18 was as an independent predictor of T2DM and it was involved in the pathogenesis, development and progression of T2DM. Besides, IL-18 may be a useful biomarker of the clinical manifestations of T2DM and for the management of the risk factors. Inflammatory cytokine IL-18 exert an important actions implicated from development of the initial stages of T2DM to progression and to late stages. So, the recognition of this molecule IL-18 as a significant pathogenic factor in this disease will provide a new therapeutic targets.

Growing evidence highlights the importance of inflammatory mechanisms in the development and progression of T2DM. Therefore, investigation the antiinflammatory strategies may offer new approaches of further effect.

The presented findings showed that the oxidative/ antioxidative status imbalance marker SOD and inflammatory markers MMp-9, IL-18 added a prognostic information regarding T2DM.

Therefore, the future studies need to focus on gathering large sample size to clarify the relationship between antioxidant depletion concomitant with inflammatory process with the incidence of T2DM. So, it was postulated that further researches on larger groups of subjects might help to elucidate the effects of hyperglycemia upon the activities SOD , of matrix metalloproteinases and IL-18 in humans.

In addition, this study offers the basis for further work on whether the manipulation of redox balance in patients with T2DM a useful approach in the design of future therapies for T2DM.

The growing knowledge and better understanding of the role of the inflammatory processes in the pathogenesis of T2DM will represent an important therapeutic opportunity for the development of new strategies that can be translated successfully into clinical applications for the treatment of T2DM.

As evidence accumulates linking inflammatory processes with T2DM, markers of inflammation may become useful by providing additional information about a patient's risk of developing T2DM, as well as providing new targets for treatment. The mechanisms of MMP-9 and IL-18 productions and activities in T2DM will allow the identification of targets for more effective therapeutic intervention.

Future studies will be aimed at preventing and /or correcting OS in T2DM by active therapeutic interventions such as by the administration of antioxidant agents contained in the diet or supplemented to it.

Further studies are needed to confirm and elucidate these findings and further prospective studies on the relationship between oxidant /antioxidant status and inflammation with the incidence of T2DM are needed to strengthen this evidence base.

There is a need to continue exploring the relationship between free radicals with T2DM to elucidate the mechanisms by which oxidative stress / antioxidant imbalance and inflammatory processes accelerate the prevalence and progression of T2DM.

\section{Abbreviation}

CRP: C-reactive protein DM: Diabetes mellitus , DNA: Deoxyribonucleic acid, ECM : Extracellular matrix , ELISA: Enzyme linked immunosorbent assay, $\mathrm{H}_{2} \mathrm{O}_{2}$ : Hydrogen peroxide, IL-18 : Interlukine-18 , MMP9 :Matrixmetalloprotinase-9, OS :Oxidative stress , ROS: Reactive Oxygen species, rpm: revolution per minutes, SOD : Superoxide dismutase, T2DM: Type 2 diabetes mellitus , TIMPs : Tissue inhibitors matrixmetalloprotinase, TH-1: T-helper-1,TNF TNF- $\alpha$, : Tumor necrosis factor TNF$\alpha$, WHO: Water Health Organization.

\section{Financial Support}

This article was partially supported by Hawler Medical University.

This article is M.Sc. thesis which was performed under my supervision at the Clinical Analysis Department, College of Pharmacy, Hawler Medical University for Degree in M.Sc. of Science in Clinical Biochemistry.

\section{References}

[1] Bisht Shradha, Sisodia S. (2010). Diabetes, Dyslipidemia, Antioxidant and Status of Oxidative Stress. International Journal of Research in Ayurveda \& Pharmacy, 1(1): 33-42 .

[2] Bigagli E., Raimondi L., Mannucci E., Colombi C., Bardini G., Rotella C.M., Lodovici1 M. (2012). Lipid and protein oxidation products, antioxidant status and vascular complications in poorly controlled type 2 diabetes. The British Journal of Diabetes \& Vascular Disease, 12: 33-39.

[3] Simmi Kharb (2010). Activity of Extracellular Superoxide Dismutase in Gestational Diabetes. Research Journal of Obstetrics and Gynecology, 3: 1-4.

[4] Dincer Y., Akcay T., Alademir Z., Ilkova H. (2002). Effect of oxidative stress on glutathione pathway in RBCs from patients with IDDM. Metabolism, 51: 1360-1362. 
[5] Jansson SP., Andersson DK., Svardsudd K. (2007). Prevalence and incidence rate of diabetes mellitus in a Swedish community during 30 years of follow-up. Diabetologia, 50(4):703- 710

[6] Ceriello A. (2000). Oxidative stress and glycemic regulation. Metabolism, 49(2 Suppl 1):27-29.

[7] Giacco F., Brownlee M. (2010). Oxidative stress and diabetic complications. Circ Res, 107:1058- 1070.

[8] Hart PJ. (2006). Pathogenic superoxide dismutase structure, folding, aggregation and turnover. Curr Opin Chem Biol, $10: 131-138$

[9] Blancas-Flores, G., Almanza-Pérez, J.C., López-Roa, R.I., Alarcón-Aguilar, F.J., García-Macedo, R.,Cruz, M. (2010). Obesity as an inflammatory process. Bol. Med. Hosp. Infant. Mex, 67: 88-96.

[10] Fernandez-Real JM, Ricart W. (2003). Insulin resistance and chronic cardiovascular inflammatory syndrome. Endocr Rev, 24: 278-301.

[11] Krzysztof C. Lewandowski, Ewa Banach, Małgorzata Bieńkiewicz, and Andrzej Lewiński, (2011). Matrix metalloproteinases in type 2 diabetes and non-diabetic controls: effects of short-term and chronic hyperglycaemia. Arch Med Sci, 7(2): 294- 303.

[12] Hopps Affiliations: Corresponding author at: Department of Internal and Specialistic Medicine, University of Palermo, Via del Vespro 129, 90127 Palermo, Italy. Tel.: +39 91655 4575; fax: + 3991655 4535/4319. E., Caimi G.(2012). Matrix metalloproteinases in metabolic syndrome. European Journal of Internal Medicine, 23 (2) : 99- 104.

[13] Chung AW, Hsiang YN, Matzke LA, McManus BM, van Breemen C, Okon EB(2006). Reduced expression of vascular endothelial growth factor paralleled with the increased angiostatin expression resulting from the upregulated activities of matrix metalloproteinase-2 and -9 in human type 2 diabetic arterial vasculature. Circ Res, 99: 140-148.

[14] Guillermo Zalba, Fortuño Ana, Díez Javier, ( 2006). Oxidative stress and atherosclerosis in early chronic kidney disease. Nephrol Dial Transplant, 21: 2686- 2690.

[15] Kreisberg J.I., Garoni J.A., Radnik R., Ayo SH. (1994). High glucose and TGF 1 stimulate fibronectin gene expression through a cAMP response element.Kidney Int, 46: 1019- 1024

[16] Okamura H., Tsutsui H., Komatsu T., Yutsudo M., Hakura A., Tanimoto T., Torigoe K., Okura T., Nukada Y., Hattori K., Akita K., Namba M., Tanabe F., Konishi K., Fukuda S., Kurimoto M. (1995). Cloning of a new cytokine that induces IFN-gamma production by T cells. Nature, 378: 88-91.

[17] Dai S.M., Matsuno H., Nakamura H., Nishioka K., Yudoh K. (2004). Interleukin-18 enhances monocyte tumor necrosis factor alpha and interleukin-1beta production induced by direct contact with $\mathrm{T}$ lymphocytes: implications in rheumatoid arthritis. Arthritis Rheum, $50: 432-443$.

[18] Guler S., Cakir B., Demirbas B., Yonem A., Odabasi E., Onde U., Aykut O., Gursoy G. (2002). Plasma soluble intercellular adhesion molecule 1 levels are increased in type 2 diabetic patients with nephropathy. Horm Res, $58: 67-70$.

[19] Dinarello C. A., Fantuzzi G. (2003) . Interleukin-18 and host defense against infection. J. Infect. Dis, $187: 370-384$.

[20] Van Guilder G.P., Hoetzer G.L., Greiner J.J., Stauffer B.L.,
Desouza C.A. (2006). Influence of metabolic syndrome on biomarkers of oxidative stress and inflammation in obese adults. Obesity Silver Spring, 14: 2127- 2131.

[21] Esposito K., Nappo F., Marfella R., Giugliano G., Giugliano F., Ciotola M., Quagliaro L., Ceriello A., Giugliano D. (2002). Inflammatory cytokine concentrations are acutely increased by hyperglycemia in humans: role of oxidative stress. Circulation, 106: 2067-2072.

[22] Netea M.G., Joosten L.A., Lewis E., Jensen D.R., Voshol P.J., Kullberg B.J., Tack C.J., van Krieken H., Kim S.H., Stalenhoef A.F., van de Loo F.A., Verschueren I., Pulawa L., Akira S., Eckel R.H., Dinarello C.A., van den Berg W., van der Meer J.W.(2006). Deficiency of interleukin-18 in mice leads to hyperphagia, obesity and insulin resistance. Nature medicine, 12(6): 650- 656 .

[23] Nakamura A., Shikata K., Hiramatsu M., Nakatou T., Kitamura T., Wada J., Itoshima T., Makino H. ( 2005). Serum interleukin-18 levels are associated with nephropathy and atherosclerosis in Japanese patients with type 2 diabetes. Diabetes Care, 28: 2890- 2895.

[24] Aldebasi Y, Mohieldein A, Almansour Y, Almoteri B (2011). Imbalance of Oxidant/Antioxidant Status and Risk Factors for Saudi Type 2 Diabetic Patients with Retinopathy. Br J Medi Med Res, 1(4): 371- 384.

[25] Lodovici M, Giovannelli L, Pitozzi V, Bigagli E, Bardini G, Rotella CM (2008). Oxidative DNA damage and plasma antioxidant capacity in type 2 diabetic patients with good and poor glycaemic control. Mutation Research, 638: 98-102.

[26] Likidlilid A, Patchanans N, Peerapatdit T (2010). Lipid peroxidation and antioxidant enzyme activities in erythrocytes of type 2 diabetic patients. Journal of the Medical Association of Thailand, 93: 682-693.

[27] Hisalkar PJ, Patne AB , Fawade MM , Karnik AC (2012). Evaluation of plasma superoxide dismutase and glutathione peroxidase in type 2 diabetic patients. Biology and Medicine, 4 (2): $65-72$.

[28] Strain J.J.( 1991). Disturbances of micronutrient and antioxidant status in diabetes. Proceedings of the Nutrition Society, 50: 591-504.

[29] Uchimura K, Nagasaka A, Hayashi R, Makino M, Nagata M, Kakizawa H, Kobayashi T, Fujiwara K, Kato T, Iwase K,Shinohara R, Kato K and Itoh M (1999). Changes in superoxide dismutase activities and concentrations and myeloperoxidase activities in leukocytes from patients with diabetes mellitus. J. Diabet. Complications, 13: 264-270.

[30] Gutteridge JM, Halliwell B (2000). Free radicals and antioxidant in the year 2000: a historical look to the future. In: Chuang ChinChiueh, ed. Reactive oxygen species from radiation to molecular biology. Annals of the New York Academy of Sciences, 899. New York: The New York Academy of Sciences:136-147.

[31] Marjani et al ,(2010). Glycoxidative stress and cardiovascular complications in experimentally-induced diabetes: effects of antioxidant treatment. Open Cardiovasc Med J, 4: 240.

[32] Ehsaneh Taheri Mahmoud Djalali, Ahmad Saedisomeolia, Ali Malekshahi Moghadam, Abolghasem Djazayeri and Mostafa Qorbani (2012). The relationship between the activates of antioxidant enzymes in red blood cells and body mass index in Iranian type 2 diabetes and healthy subjects. Journal of Diabetes \& Metabolic Disorders, 11:3 
[33] Prakash S, Sudha S (2012). Lipid Peroxidation and Antioxidant Status in South Indian Patients with Type Diabetes Mellitus . IRJP, 3(3): 132- 134.

[34] Sushma Verma, Nibha Sagar, Pushpank Vats, KN Shukla, Mohammad Abbas, Monisha Banerjee (2013). Antioxidant Enzyme Levels as Markers for Type 2 Diabetes Mellitus. International Journal of Bioassays, 2 (4) :685- 690.

[35] Opara EC (2002). Oxidative stress, micronutrients, diabetes mellitus and its complications. J R Soc Health, 122(1):28- 34.

[36] Badjatia N, Satyam A, Singh P, Seth A, Sharma A. (2009). Altered antioxidant status and lipid peroxidation in Indian patients with urothelial bladder carcinoma. J Urol Oncol, $28: 1-8$.

[37] Taniguchi N.(1992). Clinical significance of superoxide dismutases:changes in aging, diabetes, ischemia and cancer. Adv Clin Chem, 29: 1-59.

[38] Bray RC, Cockle SA, Fielden EM, Roberts PB, Rotilio G, Calabrese L. (1974) . Reduction and inactivation of superoxide dismutase by hydrogen peroxide. Biochem J, 139: 43-48.

[39] Fajans S (1995). Diabetes mellitus, definition, classification, tests. In: Endocrinology Degroat L, 3rd ed, Saunders Co, USA, : 1411- 1422 .

[40] MOUSSA S.A. (2008). Oxidative Stress in Diabetes Mellitus.Romanian J.Biophys, 18 (3) : 225-236.

[41] McLennan S.V., WangX.Y., Moreno V., Yue D.K. and TwiggS.M.(2004). Connective Tissue Growth Factor Mediates High Glucose Effects on Matrix Degradation through Tissue Inhibitor of Matrix Metalloproteinase Type 1: Implications for Diabetic Nephropathy. Endocrinology, 145 ( 12): 5646- 5655.

[42] Kowluru RA (2010). Role of matrix metalloproteinase-9 in the development of diabetic retinopathy and its regulation by $\mathrm{H}$ Ras. Invest Ophthalmol Vis Sci, 51:4320- 4326.

[43] Jieli Chen, Alex Zacharek, Yisheng Cui , Cynthia Roberts, ;Michael Chopp (2011). White Matter Damage and the Effect of Matrix Metalloproteinases in Type 2 Diabetic Mice After Stroke. Stroke, ; 42: 445- 52.

[44] Kowluru RA, Mohammad G, dos Santos JM, Zhong Q (2011). Abrogation of MMP-9 gene protects against the development of retinopathy in diabetic mice by preventing mitochondrial damage. Diabetes, 60:3023-3033.

[45] Mohammad G, Kowluru RA (2012) .Diabetic retinopathy and signaling mechanism for activation of matrix metalloproteinase-9. J Cell Physiol, 227: 1052- 1061.

[46] Karhaiya Singh ,Neeraj K.Agrawal , Sanjeerv K.Gupta, Kiram Singh (2013).A Functional Single Nucleotide Polymorphism$1562 \mathrm{C}>$ Tin the Matrix Metalloproteinase Promotor Is Associated With Type2 Diabetes an Diabetic Foot Ulcer.Internation Journal of Lower Extremely Wounds, 12(3) :199-104.

[47] Wen-Chung Tsai, Fang-Chen Liang,, Ju-Wen Cheng,, Li-Ping Lin, Shih-Chieh Chang, Hsiang-Hung Chen and Jong-Hwei S Pang (2013). High glucose concentration up-regulates the expression of matrix metalloproteinase- 9 and -13 in tendon cells. BMC Musculoskeletal Disorders, 14: 255

[48] Qing Zhong and Renu A. Kowluru (2013). Regulation of Matrix Metalloproteinase-9 by Epigenetic Modifications and the Development of Diabetic Retinopathy. Diabetes, 62 (7)
2559- 2568.

[49] Sourav Kundu , Sathnur B. Pushpakumar , Aaron Tyagi , Denise Coley , Utpal Sen (2013). Hydrogen sulfide deficiency and diabetic renal remodeling: role of matrix metalloproteinase-9.American Journal of Physiology Endocrinology and Metabolism, 304 : 1365- 1378.

[50] Chung AW, Hsiang YN, Matzke LA, McManus BM, van Breemen C, Okon EB (2006). Reduced expression of vascular endothelial growth factor paralleled with the increased angiostatin expression resulting from the upregulated activities of matrix metalloproteinase- 2 and -9 in human type 2 diabetic arterial vasculature. Circ Res, 99: 140-148.

[51] Uemura S, Matsushita H, Li W, Glassford AJ, Asagami T, Lee KH (2001). Diabetes mellitus enhances vascular matrix metalloproteinase activity: role of oxidative stress. Circ Res, 88: 1291- 1298.

[52] Vera Portik-Dobos, Mark P. Anstadt ,Jimmie Hutchinson , Mary Bannan and Adviye Ergul (2002). Evidence for a Matrix Metalloproteinase Induction/Activation System in Arterial Vasculature and Decreased Synthesis and Activity in Diabetes. Diabetes, 51(10): 3063-3068.

[53] Mike Sampson, Isabel Davies, Jelena Gavrilovic, Brendan Sussams, Jackie Brown, Sian Astley and David A Hughes (2004). Plasma matrix metalloproteinases, low density lipoprotein oxidisability and soluble adhesion molecules after a glucose load in type 2 diabetes. Cardiovasc Diabetol, 3:7-14.

[54] Krzysztof C. Lewandowski, Ewa Banach, Małgorzata Bieńkiewicz, and Andrzej Lewiński (2011). Matrix metalloproteinases in type 2 diabetes and non-diabetic controls: effects of short-term and chronic hyperglycaemia. Arch Med Sci, 7(2): 294- 303.

[55] Papazafiropoulou A, Perrea D, Moyssakis I, Kokkinos A, Katsilambros N, Tentolouris N (2010). Plasma levels of MMP-2, MMP-9 and TIMP-1 are not associated with arterial stiffness in subjects with type 2 diabetes mellitus. J Diabetes Complications, 24(1):20-27.

[56] Pradhan A (2007). Obesity, metalic syndrome, and type 2 diabetes: inflammatory basis of glucose metabolic disorders. Nutr Rev, 65: 152- 156.

[57] Pradhan AD, Cook NR, Buring JE (2003). C-reactive protein is independently associated with fasting insulin in non diabetic women. Arterioscler Thromb Vas Biol, 23 (4): 650- 655.

[58] Thorand B, Kolb H, Baumert J, Koenig W, Chambless L, Meisinger C, Ilig T, Martin S, Herder C (2005). Elevated levels of interleukin-18 predict the development of type 2 diabetes: results from the MONICA/KORA Augsburg study 1984-2002. Diabetes, 54: 2932- 2938.

[59] Netea MG, Joosten LA, Lewis E, Jensen DR, Voshol PJ, Kullberg BJ, Tack CJ, van Krieken H, Kim SH, Stalenhoef AF, van de Loo FA, Verschueren I, Pulawa L, Akira S, Eckel RH, Dinarello CA, van den Berg W, van der Meer JW.(2006). Deficiency of interleukin-18 in mice leads to hyperphagia, obesity and insulin resistance. Nature medicine, 12(6):650656.

[60] Araki S, Haneda M, Koya D, Sugimoto T, Isshiki K, ChinKanasaki M, Uzu T, Kashiwagi A (2007). Predictive impact of elevated serum level of IL-18 for early renal dysfunction in type 2 diabetes: an observational follow-up study. Diabetologia, 50:867- 873 . 
[61] Juliet Evans, Malcolm Collins, Courtney Jennings, Lize van der Merwe , Ingegerd Söderström , Tommy Olsson, Naomi S Levitt1, Estelle V Lambert and Julia H Goedecke (2007). The association of interleukin-18 genotype and serum levels with metabolic risk factors for cardiovascular disease. Eur J Endocrinol, 157: 633 - 640.

[62] Zilverschoon GR, Tack CJ, Joosten LA, Kullberg BJ, Meer JW, Netea MG (2008). Interleukin-18 resistance in patients with obesity and type 2 diabetes mellitus. Int J Obes (Lond), 32:1407- 1414

[63] Hivert MF, Sun Q, Shrader P, Mantzoros CS, Meigs JB, Hu FB (2009). Circulating IL-18 and the risk of type 2 diabetes in women. Diabetologia, 52: 2101- 2108.

[64] Jagannathan, M., McDonnell, M., Liang, Y., Hasturk, H., Hetzel, J., Rubin, D., Kantarci, A., Van Dyke, T.E., GanleyLeal, L.M., Nikolajczyk, B.S., (2010). Toll-like receptors regulate $\mathrm{B}$ cell cytokine production in patients with diabetes. Diabetologia, 53: 1461- 1471.

[65] Arnalich F, Hernanz A, Lopez-Maderuelo D, Pena JM, Camacho J, Madero R, Vazquez JJ, Montiel C (2000). Enhanced acute-phase response and oxidative stress in older adults with type II diabetes. Horm Metab Res, $32: 407$ - 412.

[66] Hung J, McQuillan BM, Chapman CM, Thompson PL, Beilby JP (2005). Elevated interleukin-18 levels are associated with the metabolic syndrome independent of obesity and insulin resistance. Arterioscler Thromb Vasc Biol, 25:1268- 1273.

[67] Meneilly GS, Tessier D (2001). Diabetes in elderly adults. J Gerontol A Biol Sci Med Sci, 56: 5-13.

[68] Molham Al-Habori,Mohamed Al-Mamari , Ali Al-Meeri (2004). Type II Diabetes Mellitus and impaired glucose tolerance in Yemen: prevalence, associated metabolic changes and risk factors. Diabetes Research and Clinical Practice, 65 (3) : 275-281.

[69] Undurti N. Das (2008). Risk of type 2 diabetes mellitus in those with hypertension. Eur Heart J, 29 (7): 952- 953.

[70] Hamilton CA, Brosnan MJ, McIntyre M,Graham D, Dominiczak AF.(2001). Superoxide excess in hypertension and aging: a common cause of endothelial dysfunction. Hypertension, 37:529-534. (2 part 2).

[71] Neil, H. A., Gatling, W., Mather, H. M., Thompson A.V. , Thorogood M., Fowler G.H., Hill R.D. and Mann J.I. (1987). The Oxford Community Diabetes Study: evidence for an increase in the prevalence of known diabetes in Great Britain. Diabetic Medicine, 4 (6) : 539-543.

[72] Ruiz C, Alegria A, Barbera R, Farre R and Lagrda MJ (1999). Lipid peroxidation and antioxidant enzymes activities in patients with type I diabetes mellitus. Scand J Clin Lab Invest, 59: 99- 105. 